\title{
Nonoperative thoracic duct embolization for traumatic thoracic duct leak: Experience in 109 patients
}

\author{
Maxim Itkin, MD, ${ }^{\mathrm{a}}$ John C. Kucharczuk, MD, ${ }^{\mathrm{a}}$ Andrew Kwak, MD, ${ }^{\mathrm{a}}$ Scott O. Trerotola, MD, ${ }^{\mathrm{a}}$ and \\ Larry R. Kaiser, MD ${ }^{\mathrm{b}}$
}

Objective: To demonstrate the efficacy of a minimally invasive, nonoperative, catheter-based approach to the treatment of traumatic chyle leak.

\begin{abstract}
Methods: A retrospective review of 109 patients was conducted to assess the efficacy of thoracic duct embolization or interruption for the treatment of high-output chyle leak caused by injury to the thoracic duct.

Results: A total of 106 patients presented with chylothorax, 1 patient presented with chylopericardium, and 2 patients presented with cervical lymphocele. Twenty patients $(18 \%)$ had previous failed thoracic duct ligation. In 108 of 109 patients, a lymphangiogram was successful. Catheterization of the thoracic duct was achieved in 73 patients $(67 \%)$. In 71 of these 73 patients, embolization of the thoracic duct was performed. Endovascular coils or liquid embolic agent was used to occlude the thoracic duct. In 18 of 33 cases of unsuccessful catheterization, thoracic duct needle interruption was attempted below the diaphragm. Resolution of the chyle leak was observed in 64 of 71 patients $(90 \%)$ post-embolization. Needle interruption of the thoracic duct was successful in 13 of 18 patients $(72 \%)$. In 17 of the 20 patients who had previous attempts at thoracic duct ligation, embolization or interruption was attempted and successful in $15(88 \%)$. The overall success rate for the entire series was $71 \%$ (77/109). There were 3 (3\%) minor complications.
\end{abstract}

Conclusion: Catheter embolization or needle interruption of the thoracic duct is safe, feasible, and successful in eliminating a high-output chyle leak in the majority $(71 \%)$ of patients. This minimally invasive, although technically challenging, procedure should be the initial approach for the treatment of a traumatic chylothorax.

(J Thorac Cardiovasc Surg 2010;139:584-90)

Posttraumatic chylothorax remains a significant complication of thoracic, cardiac, and neck surgery. Chylothorax is a particularly difficult complication to manage after esophagectomy. ${ }^{1}$ Traditionally, low-output chylothorax $(<1000$ $\mathrm{mL} / \mathrm{d}$ ) is treated conservatively with total parental nutrition or a medium-chain fatty acid diet. ${ }^{2}$ High-output chylothorax usually mandates early surgical ligation via an open or video technique. ${ }^{3,4}$ The mortality after conservative treatment of chylothorax approaches $50 \%$ but decreases to $10 \%$ after surgical repair. ${ }^{5,6}$ Recently, earlier intervention has been recommended to reduce mortality and morbidity. ${ }^{4}$

Percutaneous treatment of chylothorax was developed as a minimally invasive alternative to surgical thoracic duct ligation. ${ }^{7}$ The treatment consists of diagnostic pedal lymphangiography followed by transabdominal catheterization of the cisterna chyli/thoracic duct and embolization of the

From the Hospital of the University of Pennsylvania, ${ }^{\text {a }}$ Philadelphia, Pa; and The University of Texas, ${ }^{\mathrm{b}}$ Houston, Tex.

Disclosures: None.

Received for publication May 20, 2009; revisions received Oct 12, 2009; accepted for publication Nov 4, 2009; available ahead of print Dec 30, 2009.

Address for reprints: Maxim Itkin, MD, Hospital of University of Pennsylvania, Interventional Radiology, 3400 Spruce St, Philadelphia, PA 19004 (E-mail: itkinmax@ uphs.upenn.edu)

0022-5223/\$36.00

Published by Elsevier Inc. on behalf of The American Association for Thoracic Surgery doi:10.1016/j.jtcvs.2009.11.025 thoracic duct proximal to the chyle leak. In addition to the minimally invasive nature of the procedure, the ability to identify chyle leak and variations in thoracic duct anatomy potentially improves the outcome. In the largest series of 42 patients, Cope and $\mathrm{Kaiser}^{7}$ reported $73.8 \%$ response rates after thoracic duct embolization (TDE) and needle interruption with no morbidity or mortality. The objective of this study is to summarize our latest experience with this procedure as a treatment for traumatic chylothorax. Because of significant differences in pathophysiology, diagnosis, treatment, and outcome between nontraumatic and traumatic chylothorax, we believe that these 2 distinct entities should be reported, considered, and analyzed separately.

\section{MATERIALS AND METHODS}

Institutional review board approval was obtained for this retrospective review, and a Health Insurance Portability and Accountability Act waiver was granted. Between 1996 and early 2009, 109 patients with chyle leaks caused by iatrogenic injury were treated with TDE or thoracic duct interruption. Patient age, gender, location of the chylous effusion, treatment outcome, and complications were collected retrospectively from hospital databases and our quality assurance database (HI-IQ, Conexsys, Woonsocket, RI).

All patients had the typical "milky", appearance of chylous effusion obtained from drainage tubes or pleural tap. To confirm the diagnosis, the levels of triglycerides and cholesterol were measured and defined as diagnostic when the triglyceride level was more than $110 \mathrm{mg} / \mathrm{dL}$ and the cholesterol level was less than blood level. ${ }^{8}$ 


\section{Abbreviations and Acronyms}

$\mathrm{TDE}=$ thoracic duct embolization

Procedures were performed under moderate sedation. Prophylactic intravenous antibiotics (cefazolin) were administered before the procedure. Diagnostic pedal lymphangiography was performed. To opacify a pedal lymphatic vessel and facilitate lymphatic vessel dissection, Isosulfan blue $1 \%$ (Lymphazurin; US Surgical, Norwalk, Conn) mixed with lidocaine $1 \%$ was injected into the soft tissue of the web between the toes. After several minutes, through a small horizontal incision on the dorsum of the foot, a lymphatic duct was dissected free and cannulated using a 30-gauge needle (Cook Inc, Bloomington, Ind). Ethiodized oil (Ethiodol, Savage Laboratories, Melville, NY) was then injected through the needle using a dedicated lymphangiogram pump (Cordis, Johnson and Johnson, Miami Lakes, Fla).

The amount of contrast injected depended on physician preference but was generally not more than $15 \mathrm{~mL}$. If the cisterna chyli could not be opacified at the completion of the injection, $20 \mathrm{~mL}$ of normal saline was injected into the lymphatic vessels after the Ethiodol to propel the contrast through the lymphatic vessels and facilitate opacification of the thoracic duct and cisterna chyli (a so-called push lymphangiogram).

After identification, the target vessel (cisterna chyli, thoracic duct, or one of the tributaries) was accessed percutaneously using a 21- or 22-gauge 15to 20-cm Chiba needle (Cook Inc) via a transabdominal approach (Figure 1). Efforts were made to avoid access through the large intestine or aorta. Because of the absence of a suitable target in the upper abdomen, access was achieved retrograde in 1 patient via direct puncture of the cervical portion of the thoracic duct.

A stiff 0.018-inch guidewire (V18 Control, Boston Scientific, Natick, MA) was advanced through the needle into the cisterna chyli and further into the thoracic duct (Figure 2). A microcatheter (Rapid Transit, Cordis) $65 \mathrm{~cm}$ in length was advanced into the thoracic duct over the wire. Iodinated water-soluble contrast was injected through the microcatheter to define the leak (Figure 3). After identification of extravasation, embolization of the thoracic duct below the leak was performed. Over the course of the study period, different embolization materials were used to occlude the thoracic duct. Embolization coils initially were used to pack the lumen of the thoracic duct and impede flow. Later, with the availability of glue (TrueFill; Cordis) and Onyx (Micro Therapeutics Inc, Irvine, Calif), these liquid agents alone or in combination with intravascular coils were used (Figure 4). The TrueFill glue was diluted 1:2.5 with Ethiodol to delay polymerization; Onyx was used according to the manufacturer's instructions. Immediately after glue injection, the microcatheter was removed. If catheterization of the cisterna chyli/thoracic duct was unsuccessful, needle interruption of the thoracic duct below the diaphragm was performed using a previously described technique. $^{7}$

After the procedure, the output from drainage tubes was monitored, and if it decreased significantly, a food challenge test (ice cream or cream) was prescribed. If negative, the diet was advanced and the chest drains were removed. In patients with a "slow leak" or without a chest tube, follow-up chest radiography was used to monitor the success of the treatment. If embolization was technically successful, but failed clinically, repeat embolization was performed. In such cases, a repeat lymphangiogram was not performed, because the glue/retained contrast was used to guide the access into the cisterna chyli.

\section{RESULTS}

Overall, 109 patients (54 male, 55 female, average age 59 years) were treated over a period of 13 years, 40 of whom

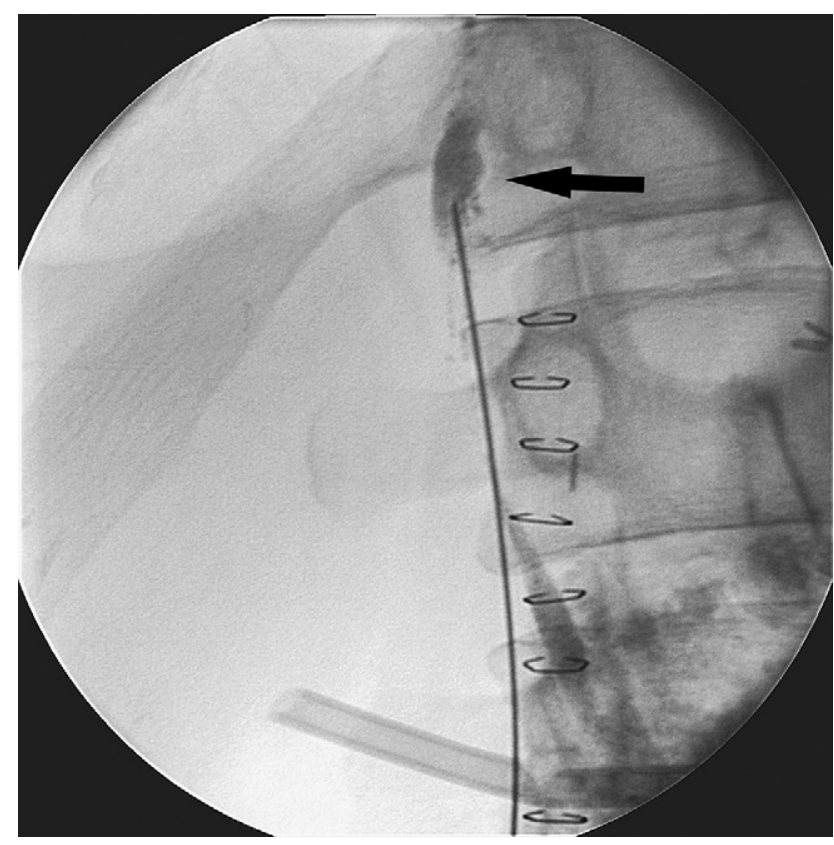

FIGURE 1. Radiographic image showing attempt to access cysterna chyli opacified with contrast (arrow) with 21-gauge needle.

were referred from the outside facilities. A total of $106 \mathrm{pa}-$ tients presented with chylothorax, 1 patient presented with chylopericardium, and 2 patients presented with chylous leaks in the subcutaneous tissue of the neck. Among patients with chylothorax, 47 of $106(44 \%)$ presented with left effusions, 46 of $106(43 \%)$ presented with right effusions, and 13 of $106(13 \%)$ presented with bilateral effusions. The causes of the chylous leak are listed in Table 1 . Twenty of 109 patients $(18 \%)$ presented with previously unsuccessful thoracic duct ligation.

Lymphangiography was successful in all but 1 patient $(108 / 109,99 \%)$. In 2 of 108 patients, catheterization was deemed impossible because of unsuitable anatomy and was not attempted. In 73 of 106 patients (67\%), catheterization of the thoracic duct was successful. After successful catheterization, TDE was performed in 71 of 73 patients. In 2 patients in whom catheterization was successful, embolization was not attempted because of the observation of a successful (surgical) thoracic duct ligation. Later, we learned that a radiographically successful ligation does not imply clinical success and that even in such cases, embolization of the cisterna chyli could result in clinical resolution of the chylothorax.

Embolization was performed in 13 of 71 patients (18.3\%) with coils only; in 18 of 71 patients $(25.4 \%)$ with liquid embolic agents; and in 40 of 71 patients $(56.3 \%)$ with a combination of liquid embolic agents and coils. In 18 of 33 cases of unsuccessful catheterization, thoracic duct interruption was performed. 


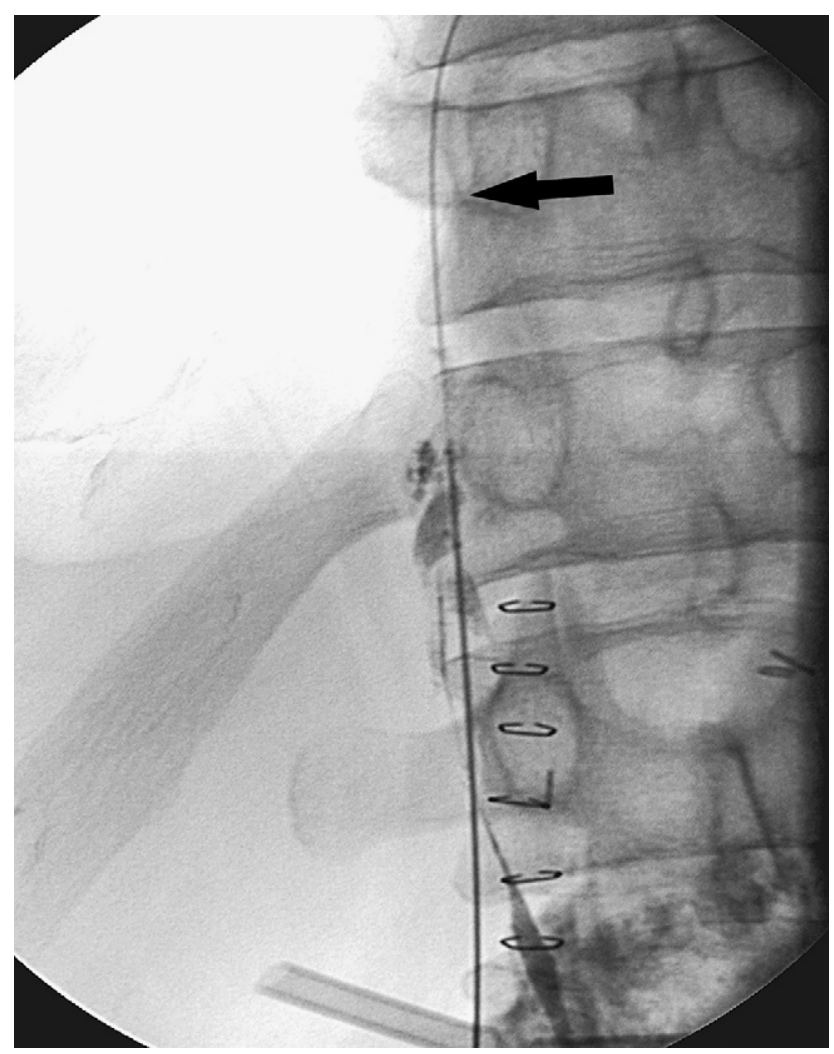

FIGURE 2. Radiographic image showing V18 wire (arrow) in the cysterna chyli and thoracic duct.

Follow-up was available in all but 2 patients. The overall success rate for the entire series on intent-to-treat basis was 77 of 109 patients $(71 \%)$. The overall success in the subgroup of patients with attempted interventions (excluding patients in whom catheterization was not attempted $[\mathrm{n}=2]$, embolization was not attempted [ $\mathrm{n}=2]$, and interruption was not attempted $[n=17])$ was 77 of 88 patients $(88 \%)$.

Clinical success after technically successful TDE was 64 of 71 patients $(90 \%$ ) (4 patients with failures, 2 patients were lost to follow-up, and 1 patient died within several days post-procedure from unrelated causes). In 6 patients, embolization was performed twice, after an initial unsuccessful attempt. In all 6 patients, the second embolization attempt was successful. The causes of failure were inadequate filling of the lumen of the thoracic duct with liquid embolic agent or coils.

In patients in whom liquid embolic agents were used, the success of embolization was 53 of 58 patients $(91 \%)$ (2 patients with failures, 2 patients with no follow-up, and 1 patient died within 1 week after embolization). The success rate for coil only embolization was 11 of 13 patients $(84 \%)$. The clinical success of thoracic duct needle interruption was 13 of 18 patients $(72 \%)$.

In the subgroup with failed thoracic duct ligation, TDE was attempted in 17 of 20 patients. Intervention was not at-

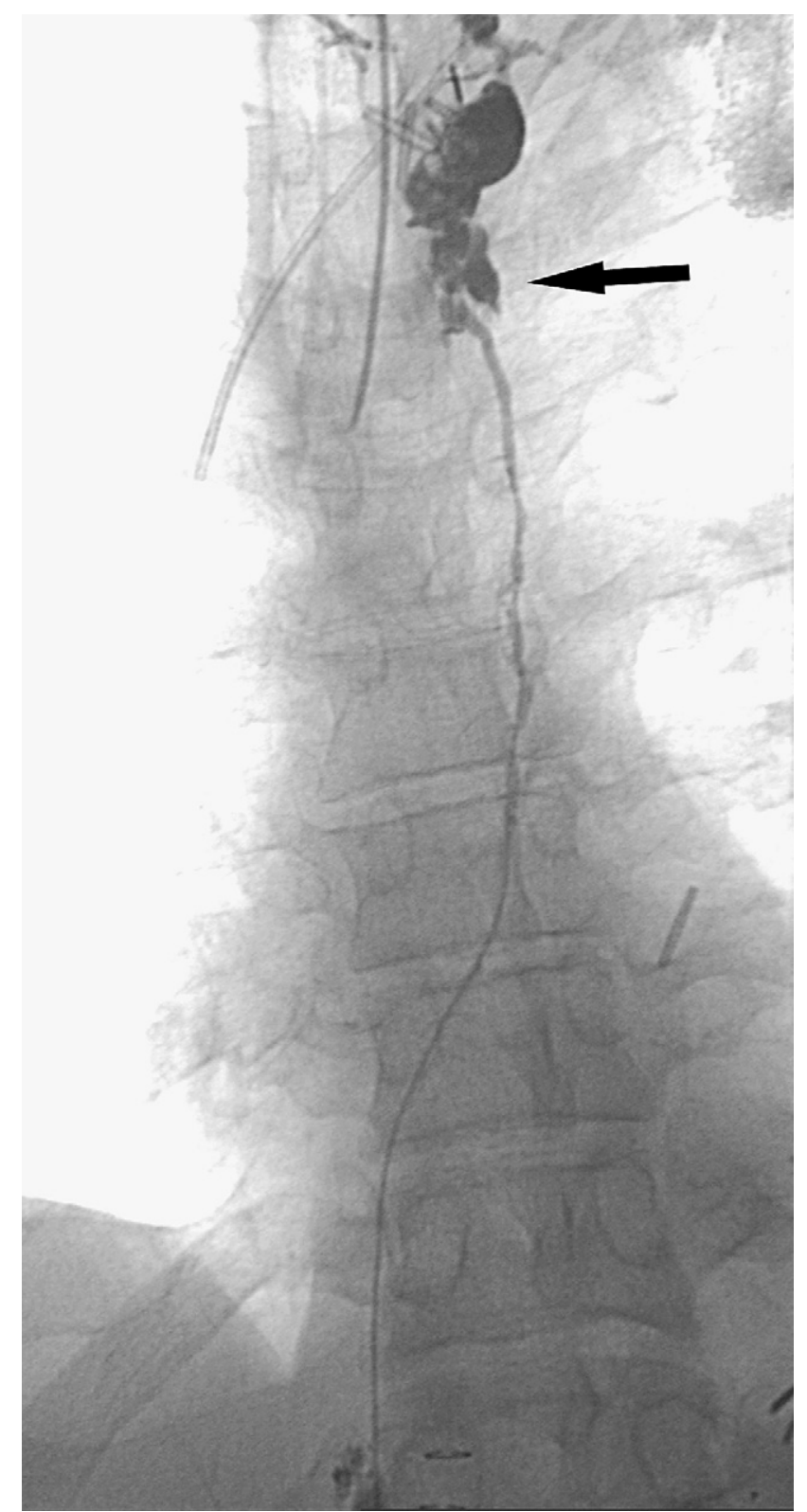

FIGURE 3. Radiographic image showing extravasation of the contrast in the upper part of the thoracic duct (arrow), following injection of the duct with iodinated contrast through the microcathetr.

tempted because of the radiographic appearance of the occluded cisterna chyli (1 patient with no catheterization attempt and 2 patients with successful catheterizations, no embolization attempt). As mentioned previously, these cases were treated in the early investigational stages of this procedure, and latter similar cases treated by embolization resulted in cure. In this setting, embolization was successful in 15 of 17 patients $(88 \%)$.

There were 3 complications related to the procedure: 1 case of asymptomatic embolization of the pulmonary artery with glue and 2 cases of leg edema and pedal suture 


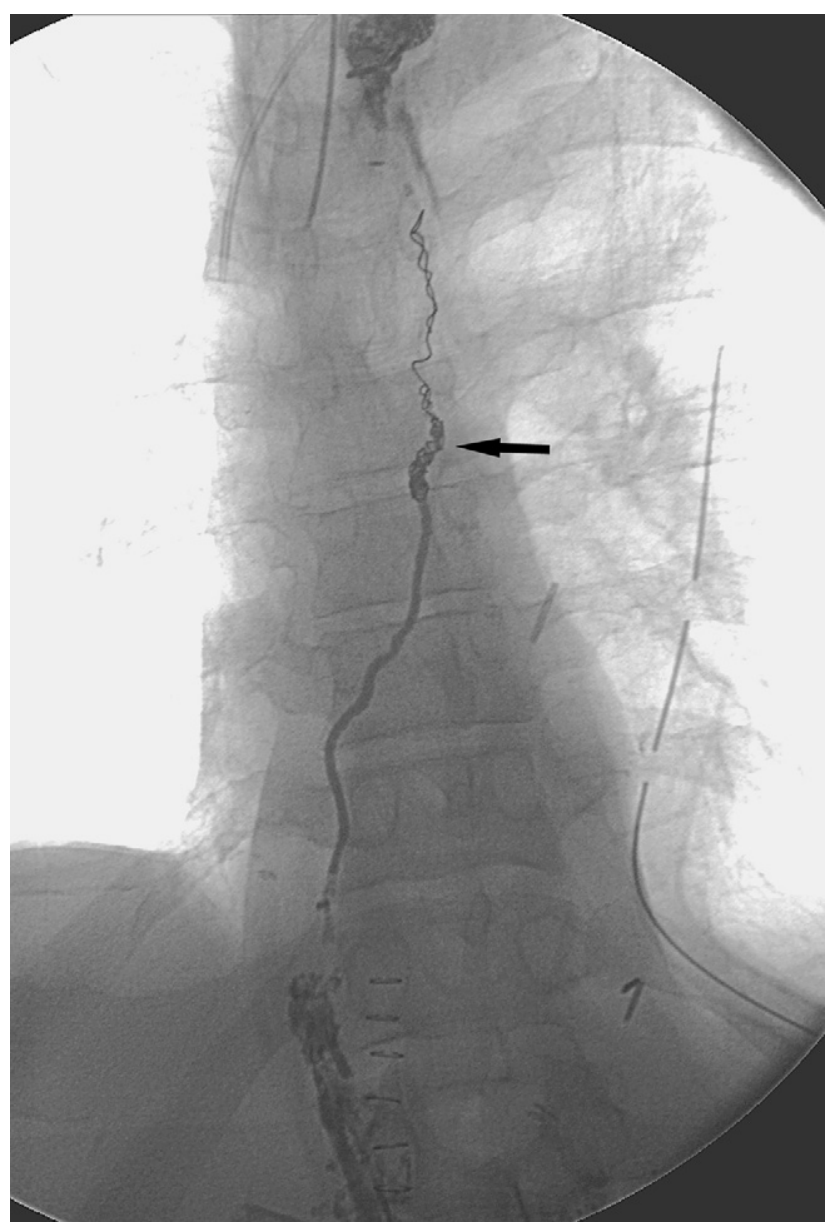

FIGURE 4. Radiographic image showing the occluded thoracic duct filled with coils (arrow) and glue cast below the colis.

dehiscence that resulted in would infections. The leg edema eventually subsided, and the infections were cured with local care and antibiotics.

\section{DISCUSSION}

Chylous effusion after operations involving the mediastinum and neck is a rare but serious complication with significant mortality and morbidity. It has been reported to occur at a rate of $0.42 \%$ for all general thoracic surgery procedures $^{9}$ and up to $3.9 \%$ after esophagectomy. ${ }^{1}$ Traditionally, low-output chylothorax $(<1000 \mathrm{~mL} / \mathrm{d})$ is treated conservatively by complete cessation of oral intake and administration of total parenteral nutrition or a medium-chain triglycerides diet, ${ }^{9}$ and operative intervention is suggested for cases with failure. In cases of high-output chylothorax $(>1000 \mathrm{~mL} / \mathrm{d})$, immediate reoperation with thoracic duct ligation has been recommended. ${ }^{9-11}$ The mortality after conservative treatment of chylothorax approaches $50 \%$ and can reach $10 \%{ }^{5,6}$ or $16 \%$ after surgical repair in high-risk patients. ${ }^{12}$ Early intervention to stop chyle leakage has been advocated recently to reduce perioperative mortality
TABLE 1. Causes of traumatic chylous leak

\begin{tabular}{lr}
\hline Esophagectomy & 31 \\
Lung resection for cancer & 29 \\
Coronary bypass & 9 \\
Thoracic aneurysm repair & 6 \\
Heart transplant & 4 \\
Trauma & 4 \\
Lung transplant & 3 \\
Aortic valve replacement & 2 \\
Mediastinal biopsy & 2 \\
Mediastinal mass resection & 2 \\
Cardiac tumor resection & 2 \\
Thyroidectomy & 2 \\
AAA repair & 1 \\
Aortic dissection repair & 1 \\
Aortic transection repair & 1 \\
Back surgery & 1 \\
Diaphragmatic hernia repair & 1 \\
Mitral valve replacement & 1 \\
Neck dissection for cancer & 1 \\
Neck dissection for lipoma & 1 \\
PDA ligations & 1 \\
Pericardial surgery & 1 \\
Radiation & 1 \\
Rib resection for thoracic outlet syndrome & 1 \\
Thymomectomy & 1 \\
Total & 1 \\
\hline AAA Abdral & 1 \\
\hline
\end{tabular}

$A A A$, Abdominal aortic aneurysm; $P D A$, patent ductus arteriosus.

and morbidity. ${ }^{4}$ Indeed, prolonged leakage of chyle, rich in nutrients, T cells, and electrolytes, in the early postoperative period can significantly worsen a patient's condition. ${ }^{9}$

This report represents our latest experience with TDE and thoracic duct needle interruption for traumatic chylothorax. The procedure was designed and developed over the years by Cope and Kaiser ${ }^{7}$ and Cope. ${ }^{13-15}$ Since its original description, several authors have reported their experience in case reports and small series. ${ }^{16-21}$ The rationale for the procedure is to improve the success rate of thoracic duct interruption by using lymphangiography as a "road map" and to reduce perioperative mortality and morbidity by using a percutaneous, minimally invasive approach.

Lymphangiography has been used extensively to diagnose chylous leaks and define thoracic ductal anatomy before operation and especially in cases of failed thoracic duct ligation. ${ }^{9,22-24}$ In our series, 20 patients were referred after failed surgical thoracic duct ligation. In all of these cases, lymphangiography could identify and guide treatment that was successful in $90 \%$ of the subgroup in whom intervention was attempted. Even in cases of failed TDE, the ability to identify the leak and underlying thoracic duct anatomy facilitated and guided a subsequent surgical approach.

After initial encouraging reports of high success, low complication rates of TDE/needle interruption, ${ }^{7}$ and a plea for "early thoracic duct intervention," ${ }^{, 4}$ patients are referred 
for embolization within a few days of diagnosis of chylothorax at the Hospital of the University of Pennsylvania, without distinction between high and low output.

The minimally invasive nature of the procedure is reflected in significantly lower morbidity and mortality after TDE. In the original surgical series, mortality and morbidity rates were $2.1 \%$ and $38 \%$, respectively. ${ }^{9}$ We observed only 3 minor complications of the procedure in this study. This correlates well with previous reports by Cope and Kaiser, ${ }^{7}$ who reported no complications, and Boffa and colleagues, ${ }^{21}$ who reported 1 case of bile leakage. The latter group used a transhepatic approach and a larger 4F cannula to access the cisterna chyli. We think that a transabdominal approach with 21- to 22-gauge Chiba needles followed by 3F microcatheters represents a safer option. We did not observe any complication related to transabdominal access, even though it was through all upper abdominal structures. An additional advantage of the procedure is the ability to perform it under local anesthesia and moderate sedation without general anesthesia and single-lung ventilation. In some cases, TDE was performed as a 1-day admission procedure.

One of the limitations of this study is its retrospective nature; thus, it is possible that a few minor complications were missed because some patients were transferred to our facility for the procedure only and the clinical follow-up was performed in the outside hospital. However, we believe that we would be aware of significant complications related to the procedure had they occurred

The overall success rate (intent to treat) in our series was $71 \%$, which is comparable to $73.5 \%$ (partial and complete response) reported by Cope and Kaiser ${ }^{7}$ and better than $45 \%$ reported by Boffa and colleagues. ${ }^{21}$ The slightly lower success rate compared with that reported by Cope and Kaiser could be explained by the fact that the intervention was attempted in only 88 of $109(80 \%)$ of our patients. Of note, in the patient subgroup with attempted interventions the success rate was 77 of 88 patients $(88 \%)$.

The success rate is directly related to the ability to catheterize the cisterna chyli/thoracic duct; when the catheterization was successful, the cure rate was $90 \%$. Several factors affect the success of catheterization, including the ability to visualize the cisterna chyli/thoracic duct, the patient's body habitus, and operator experience. Because of high flow in the intestinal lymphatics, which constitutes $80 \%$ of the lymphatic flow in the thoracic duct, contrast that reaches the cisterna chyli through the lumbar lymphatics becomes diluted at the level of their convergence. To deliver a significant bolus of contrast to the area of cisterna chyli, later in our experience, we followed the injection of contrast with a saline bolus (in patients in whom the target could not be identified at the end of contrast injection). This was to propel intralymphatic contrast material further to the level of cisterna chyli and to improve identification of the target vessel. The overall success of catheterization was $67 \%$ in our
Catheterization success over the years

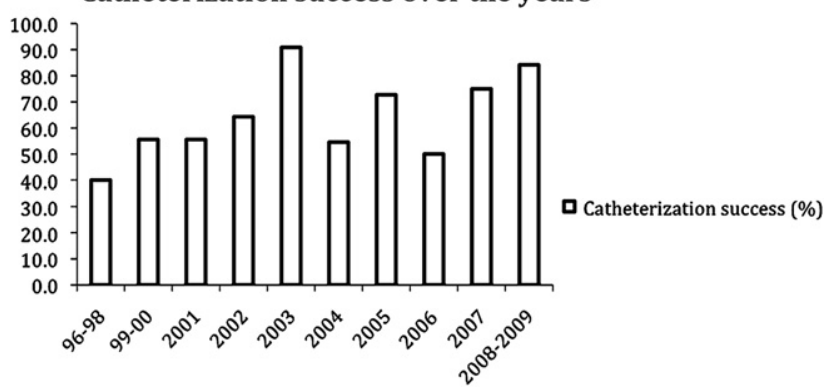

FIGURE 5. Catheterization success of the cisterna chyli over the years.

series and $62 \%$ in Cope and Kaiser's ${ }^{7}$ report. Operator experience plays a significant role in the success of the cisterna chyli catheterization, which is reflected in Figure 5. Up to 2003 , a single operator performed the bulk of the procedures, and the success rate gradually reached $91 \%$. New operators started to perform the procedure in 2003, and the success rate decreased to $54 \%$ and slowly increased to $84 \%$ in 2008 and 2009.

Although we see the thoracic duct interruption as a "last resort" in case of failed thoracic duct catheterization, it was proven to be somewhat effective, resulting in resolution in $72 \%$ of cases in our series, which is comparable to the $43 \%$ reported by Cope and Kaiser. ${ }^{7}$ Lymphangiography itself can result in cessation of chylothorax, as was reported by Matsumoto and colleagues. ${ }^{25}$

We strongly believe that use of a combination of coils and liquid embolic agent is necessary to achieve safe and adequate closure of the thoracic duct. Coils serve as a matrix for glue polymerization and provide an additional safety measure in case of glue failure. Embolization with coils alone results in higher failure rates when compared with liquid embolic agents ( $84 \%$ vs $91 \%$ ).

Two patients who were referred from the outside facility were lost to follow-up, which is the major limitation of this study.

\section{CONCLUSIONS}

Percutaneous TDE and needle interruption should be the initial treatment of choice for posttraumatic chylous effusion because there is a high success rate and minimal complications. This constellation of interventions does require experience in thoracic duct catheterization and expertise in performing lymphangiography. The latter procedure is not routinely performed in most centers, and the skills required are disappearing. In addition, dedicated lymphangiogram pumps are not manufactured any longer and the operators need to rely on substitute equipment, such as the Harvard pump (Harvard Apparatus, Holliston, Mass). As an alternative, ultrasound-guided groin lymph node injection could be performed to opacify the cisterna chyli and thoracic duct (unpublished data). We hope that with the development of 
additional lymphatic procedures, the demand for lymphangiography will increase and the skills will be redeveloped. We continue to work to improve the technical success that is directly related to the ability to visualize and cannulate the cisterna chyli and thoracic duct. Visualization of the thoracic duct has improved significantly since we started to use the saline flush (push lymphangiogram). The reverse Trendelenburg position of a patient may further improve cisterna chyli opacification using gravity to slow the flow in the thoracic duct. ${ }^{23}$ From our limited experience, the retrograde transvenous or direct approach through the subclavian vein facilitates catheterization of the thoracic duct and seems to be less technically challenging.

\section{References}

1. Dougenis D, Walker WS, Cameron EW, Walbaum PR. Management of chylothorax complicating extensive esophageal resection. Surg Gynecol Obstet. 1992;17:501-6.

2. Marts BC, Naunheim KS, Fiore AC, Pennington DG. Conservative versus surgical management of chylothorax. Am J Surg. 1992;164:532-5.

3. Christodoulou M, Ris HB, Pezzetta E. Video-assisted right supradiaphragmatic thoracic duct ligation for non-traumatic recurrent chylothorax. Eur J Cardiothorac Surg. 2006;29:810-4.

4. Merigliano S, Molena D, Ruol A, Zaninotto G, Cagol M, Scappin S, et al. Chylothorax complicating esophagectomy for cancer: a plea for early thoracic duct ligation. J Thorac Cardiovasc Surg. 2000;119:453-7.

5. Bolger C, Walsh TN, Tanner WA, Keeling P, Hennessy TP. Chylothorax after oesophagectomy. Br J Surg. 1991;78:587-8.

6. Orringer MB, Bluett M, Deeb GM. Aggressive treatment of chylothorax complicating transhiatal esophagectomy without thoracotomy. Surgery. 1988;104:720-6.

7. Cope C, Kaiser LR. Management of unremitting chylothorax by percutaneous embolization and blockage of retroperitoneal lymphatic vessels in 42 patients. $J$ Vasc Interv Radiol. 2002;13:1139-48.

8. Staats BA, Ellefson RD, Budahn LL, Dines DE, Prakash UB, Offord K. The lipoprotein profile of chylous and nonchylous pleural effusions. Mayo Clin Proc. 1980;55:700-4.

9. Cerfolio RJ, Allen MS, Deschamps C, Trastek VF, Pairolero PC. Postoperative chylothorax. J Thorac Cardiovasc Surg. 1996;112:1361-6.

10. Patterson GA, Todd TR, Delarue NC, Ilves R, Pearson FG, Cooper JD. Supradiaphragmatic ligation of the thoracic duct in intractable chylous fistula. Ann Thorac Surg. 1981;32:44-9.

11. Selle JG, Snyder WH, Schreiber JT. Chylothorax: indications for surgery. Ann Surg. 1973; 177:245-9.

12. Sieczka EM, Harvey JC. Early thoracic duct ligation for postoperative chylothorax. J Surg Oncol. 1996;61:56-60.

13. Cope C. Percutaneous thoracic duct cannulation: feasibility study in swine. J Vasc Interv Radiol. 1995;6:559-64.

14. Cope C. Percutaneous transabdominal embolization of thoracic duct lacerations in animals. J Vasc Interv Radiol. 1996;7:725-31.

15. Cope C. Diagnosis and treatment of postoperative chyle leakage via percutaneous transabdominal catheterization of the cisterna chyli: a preliminary study. $J$ Vasc Interv Radiol. 1998;9:727-34.

16. Bonn J, Sperling D, Walinsky P, Mannion J. Percutaneous embolization of thoracic duct injury. Circulation. 2000;102:268-9.

17. van Goor AT, Kröger R, Klomp HM, de Jong MA, van den Brekel MW, Balm AJ. Introduction of lymphangiography and percutaneous embolization of the thoracic duct in a stepwise approach to the management of chylous fistulas. Head Neck. 2007;29:1017-23.

18. Schild H, Hirner A. Percutaneous translymphatic thoracic duct embolization for treatment of chylothorax. Fortschr Geb Röntgenstr Nuklearmed. 2001;173:580-2.

19. Patel N, Lewandowski R, Bove M, Nemcek A, Salem R. Thoracic duct embolization: a new treatment for massive leak after neck dissection. Laryngoscope. 2008; 118:680-3.

20. Litherland B, Given M, Lyon S. Percutaneous radiological management of highoutput chylothorax with CT-guided needle disruption. J Med Imaging Radiat Oncol. 2008;52:164-7.
21. Boffa D, Sands M, Rice T, Murthy S, Mason D, Geisinger M, et al. A critical evaluation of a percutaneous diagnostic and treatment strategy for chylothorax after thoracic surgery. Eur J Cardiothorac Surg. 2008;33:435-9.

22. Sachs PB, Zelch MG, Rice T, Geisinger MA, Risius B, Lammert GK. Diagnosis and localization of laceration of the thoracic duct: usefulness of lymphangiography and CT. AJR Am J Roentgenol. 1991;157:703-5.

23. Schulman A, Fataar S, Dalrymple R, Tidbury I. The lymphographic anatomy of chylothorax. Br J Radiol. 1978;51:420-7.

24. Ngan H, Fok M, Wong J. The role of lymphography in chylothorax following thoracic surgery. Br J Radiol. 1988;61:1032-6.

25. Matsumoto T, Yamagami T, Kato T, Hirota T, Yoshimatsu R, Masunami T, et al The effectiveness of lymphangiography as as treatment method for various chyle leakages. Br J Radiol. 2009;82:286-90. Epub 2008 Nov 24.

\section{Discussion}

Dr N. Altorki (New York, NY). I have no disclosures. Dr Itkin, I want to congratulate your group, but especially Dr Cope, for developing this in 1998, perfecting it, and really advocating it. For decades the treatment of choice for high-volume chylous fistula has been surgical ligation of the thoracic duct, and over the years several important lessons have been learned. Foremost among these lessons is the need for prompt return to the operating room, usually within 7 or no more than 10 days from the index procedure, to avoid the deleterious effects of nutritional and immunologic depletion.

Second, it is critical that surgeons involved in the treatment of chylous fistula have an intimate knowledge of thoracic duct anatomy because only precise identification and ligation of the duct will work. In a few cases, video-assisted techniques are ideally suited for that procedure. The cumulative effect of that is a success rate in excess of $95 \%$ and a procedure-related mortality of less than $1 \%$. There are only a few reasons why thoracic duct ligation does not work: The duct is not ligated, there is an accessory duct, which is rare, or there is the misfortune, as I had, of performing a mediastinal node dissection in a patient with liver cirrhosis that you did not fully appreciate. That is a big problem.

Against that backdrop we are presented with your data, which are truly impressive and noteworthy: a $71 \%$ success rate on an intention-to-treat basis and $88 \%$ on the basis of treatment actually delivered. In thoracic duct cannulation, when successful, and it is only successful two thirds of the time, the leak can be stopped in $90 \%$ of those in whom the duct is cannulated. If it is not cannulated, thoracic duct interruption by the needle is a poor alternative and also works about two thirds of the time. So what is a thoracic surgeon to do balancing the two? I think it will all depend on the available institutional expertise and the cost/benefit analysis of one versus the other, including the length of hospital stay. So my first question is, what happened to the 32 patients in whom your intervention was not successful? How were they treated and what was their outcome?

Dr Itkin. We didn't collect these data, but to my knowledge these patients underwent thoracic surgery and thoracic duct ligation was performed.

Dr Altorki. What was the outcome?

Dr Itkin. I don't have the data.

Dr Altorki. I suspect that the greatest impediment to wider adoption of this technique is not going to be the thoracic surgeon but rather the interventional radiology community. How well do you think that procedure was received by the general interventional radiology community?

Dr Itkin. Unfortunately, there is only one place I am aware of that does this procedure on a routine basis. I think the Cleveland 
Foundation published a study 2 years ago. Other than that, I get occasional reports about this procedure from my colleagues in interventional radiology. I get a weekly telephone call regarding this procedure.

The major obstacle is the lymphangiogram. The older physicians know how to do that, but they don't have the equipment, and the equipment is not currently manufactured. Right now we have a standing order on eBay to buy any lymphangiogram pump in the world. And obviously the new generation of interventional radiologists don't know how to perform a lymphangiogram.

Lymphatic intervention became my passion, so we are developing more lymphatic procedures, such as percutaneous imageguided lymphatic ligation, to treat postoperative lymphocele. In the animal laboratory we are trying to develop the technique to treat obesity with some thoracic duct intervention and T-cell depletion therapy for the bone marrow transplant recipient as a treatment for graft-versus-host disease. So I am hoping that in the near future lymphatic intervention will become more common, people will start making equipment, and physicians will start to learn how to perform a lymphangiogram.

Dr Altorki. My last question has to do with the fact that you had one asymptomatic glue embolus, and, to me, that is a harrowing thing if it occurs in the interventional radiology suite, a relatively uncontrolled situation. Have you modified your technique? Does it have to do with the amount of glue you inject? Does it have to do with whether you inject the coils first or the glue first? Has it gone up through the systemic venous circulation and down the superior vena cava, or did you just mention that it went through the inferior vena cava?

Dr Itkin. Generally, the amount of glue we inject is approximately $1.5 \mathrm{~mL}$; so if we spill one third of that, it is only $0.5 \mathrm{~mL}$ of glue. This specific case was a healthy patient who was shot in the chest through the mediastinum, and the only injured vessel was the lymphatic duct, and he could tolerate this complication well.

We have another article in process that is dedicated to nontraumatic chylous effusion (in this article, we presented only traumatic cases), and in which we describe 1 symptomatic case of pulmonary embolization with glue. The operator probably over-injected the glue into the thoracic duct. In this case, the glue spilled antegrade through the thoracic duct into the subclavian vein and then into the pulmonary circulation. In my case, the glue was spilled retrograde into the inferior vena cava and then further into the pulmonary circulation. Since then, we routinely put the first coil to create the matrix for glue polymerization and then the glue is injected below this level. But absolutely, we modified this technique. This physician was injecting glue only, but now we embolize with coils and glue simultaneously.

Dr Altorki. Thank you, and may I transfer my patients who have liver cirrhosis to you?

Dr Itkin. Not yet. The truth is, we are summarizing these data of nontraumatic chylothorax, and whenever we have the data, we will have answers.

Dr J. Cooper (Philadelphia, Pa). I came to the University of Pennsylvania and heard about this, and I can honestly tell you that I was not initially enthusiastic, but I have to say that Dr Itkin and colleagues have been a tremendous support. There are several patients, some of my own, who had an esophagectomy under cir- cumstances in which I really did not want to go back to the operating room because of postoperative problems, and his ability to deal with this in those situations has been a tremendous resource.

Max, when I first heard you discuss this, I said, no, it is not possible. I don't care how good they are, they cannot cannulate the thoracic duct. May I point out, if I am correct, and it makes it more believable, that what you do is access the cisterna chyli and then through the cisterna cannulate up to the thoracic duct. Perhaps I am wrong, but that was my understanding, and this is what allows you to have such a high rate of access, or are you actually puncturing the thoracic duct itself?

Dr Itkin. Truthfully, whatever target I see, I go after, and I don't care what it is. If it is some duct that contributes to the cisterna chyli, I will take that. Is it difficult? Yes, but as you know with experience comes success. We managed to embolize a 1-month-old child 3 weeks ago. Embolization was technically successful but partially clinically successful. We will try to repeat TDE in this child. It is challenging and definitely requires some expertise.

And, one more time, thank you for the saline flush you taught me about. It does help to see the thoracic duct better. We are thinking about different ways to improve visualization of the thoracic duct and cisterna chyli.

Dr S. Bolukbas (Wiesbaden, Germany). Do you combine your approach post-interventionally with your low-fat diet, which may lower the intraduct pressure and facilitate the closure of the thoracic duct?

Dr Itkin. If the embolization is successful, we usually see it the next day if the output drops to zero the day after the procedure. If the output is reduced but not dropped, we know that the thoracic duct is filled inadequately with glue. If it is completely unsuccessful, something went wrong with our procedure and we have to think about some other cause of chylothorax. But absolutely, a low-fat diet is a part of the arsenal of the treatment of the chylothorax.

Dr D. Sugarbaker (Boston, Mass). I congratulate you on a terrific presentation and your group for your leadership in this area. At the Brigham, we have been following your lead for several years and have found this to be a tremendous advantage for patients, particularly after pleural pneumonectomy or esophagectomy. Not having to go back or re-anesthetize the patient can often make the difference in terms of their speedy postoperative recovery. It is a tremendous advantage.

Could you comment on your initial approach? Is it with a coil in each case? Is that changing now as some of the new glues, as you mentioned, are coming along?

Dr Itkin. We have been using the same approach for the last 3 years. First, we put 2 or 3 coils just below the leak, or some other abnormality, such as collaterals, and then we inject the glue below the coils. The disadvantage of glue is that its setting time is several seconds. So injection has to be performed extremely fast. One of my colleagues started to use the Onyx embolic system, where you have complete control over the injection; it can be injected within 20 minutes. I personally still use glue because I did in almost 70 cases and feel comfortable with that. But we have to put coils before the glue. There are occasional situations, such as unsuccessful ligation to the thoracic duct, in which there is not enough space to place a coil in the thoracic duct stump. In that case you use only glue. 